\title{
Predicting Plasma Olanzapine Concentration Following a Change in Dosage: A Population Pharmacokinetic Study
}

\author{
Authors \\ T. Tsuboi ${ }^{1,2}$, R. R. Bies ${ }^{3,4,5}$, T. Suzuki ${ }^{2,6}$, H. Takeuchi ${ }^{2,7,8}$, S. Nakajima 2, 5, 8, 9, A. Graff-Guerrero ${ }^{5,8,9}$, \\ D. C. Mamo ${ }^{5,8,10}$, F. Caravaggio ${ }^{8,9}$, E. Plitman ${ }^{8,9}$, M. Mimura ${ }^{2}$, B. G. Pollock ${ }^{5,8}$, H. Uchida $^{2,5}$ \\ Affiliations \\ Affiliation addresses are listed at the end of the article
}

Key words
olanzapine
plasma concentration
population pharmaco-
kinetics
therapeutic drug monitoring

\section{Abstract \\ $\nabla$}

Introduction: Due to high inter-individual variability in peripheral pharmacokinetic parameters, dosing of antipsychotics currently relies on clinical trial-and-error, and predicting antipsychotic plasma concentrations before changing a dose has been a challenge.

Methods: Patients with schizophrenia receiving a stable dose of olanzapine were included. 2 plasma samples were collected at 2 given time points for the measurement of plasma olanzapine concentrations. At least 7 days after a dosage change of olanzapine, a third sample was collected. The plasma concentration of the third sample was predicted in a blinded fashion using a mixed-effects model with NONMEM $^{\circledR}$, using the following information: the 2 baseline plasma concentrations, the interval between the last dose and blood draw, and clinical and demographic information.

Results: 31 subjects (mean \pm SD age $=56.0 \pm 11.6$; 19 men) were enrolled. The mean prediction (95\% confidence interval) errors were $1.6(-2.8$ to 6.0$) \mathrm{ng} / \mathrm{mL}$. A highly significant correlation was observed between the observed and predicted concentrations of the third sample $(\mathrm{r}=0.91, \mathrm{p}<0.001)$.

Discussion: Plasma olanzapine concentrations following an actual dosage change can be predicted in advance with a high degree of certainty.

\section{received 28.07.2015 \\ revised $\quad 21.09 .2015$ \\ accepted $\quad 28.09 .2015$}

\section{Bibliography}

DOI http://dx.doi.org/ 10.1055/s-0035-1565070

Published online:

October 27, 2015

Pharmacopsychiatry 2015;

48: 286-291

(c) Georg Thieme Verlag KG

Stuttgart $\cdot$ New York

ISSN 0176-3679

\section{Correspondence}

H. Uchida, MD, PhD

Department of Neuropsychiatry Keio University School of Medicine

35 Shinanomachi

Shinjuku-ku

Tokyo 160-8582

Japan

hiroyuki.uchida.hu@gmail.com

\section{Introduction}

$\nabla$

Antipsychotic drugs, which exert their clinical effects mainly through the blockade of dopamine $D_{2}$ receptors [1], are widely used for the treatment of schizophrenia across the patient's life span [2]. Positron emission tomography (PET) studies have identified that optimal clinical response in young patients with schizophrenia is related to a "therapeutic window" of the striatal dopamine $D_{2}$ receptor occupancy of $65-80 \%$ $[1,3,4]$. On the other hand, the clinical application of PET to individualized antipsychotic dosing at the bedside has not been feasible because of its low availability and high cost. To tackle this situation, the authors have developed a model to estimate dopamine $D_{2}$ receptor occupancy levels from plasma concentrations of various antipsychotics, including olanzapine, with a high degree of accuracy [5]. Therefore, individualized dosing with the measurement of antipsychotic plasma concentrations could become a real potential clinical application; the current dose adjustment still relies on a trial-and-error strategy that carries a risk of relapse and adverse effects.

Given high inter-individual variability in peripheral pharmacokinetic parameters [6], the next hurdle is to accurately predict antipsychotic plasma concentrations before an actual dosage change of antipsychotic drugs for each individual. In contrast to conventional pharmacokinetic modeling, the population pharmacokinetic model can, in theory, predict individual pharmacokinetic parameters for antipsychotics, including peak and trough plasma concentrations, using blood samples collected at any given time points with the information on demographic and clinical characteristics of the subjects and dosing information (i.e., dose, time of last dose, and duration between the last dose and blood draw). Thus, the corresponding peak and trough dopamine $D_{2}$ receptor occupancy levels could also be estimated using the above-mentioned prediction model $[5,7]$. In theory, this model could be used to find doses which would result in estimated peak and trough drug concentrations corresponding to the established therapeutic window 
of dopamine $\mathrm{D}_{2}$ receptor occupancy in the treatment of schizophrenia [8]. The authors have successfully reported on the validity and reliability of predicting plasma concentrations of risperidone and 9-hydroxyrisperidone associated with a dosage change, using population pharmacokinetic techniques [9].

Olanzapine is one of the most frequently and widely used antipsychotic drugs $[10,11]$. This drug is characterized by its effect on not only psychotic but also anxiety and depressive symptoms in patients with schizophrenia $[12,13]$. Unfortunately, the use of olanzapine occasionally results in a variety of side effects, including cardiovascular and motor side effects as well as impairment in subjective well-being, some of which are at least in part dose-dependent $[3,14,15]$. Moreover, this drug may increase the risk of weight gain and diabetes mellitus in a dosedependent fashion $[16,17]$, although this dose-dependence has not always been a consistent finding in the literature $[18,19]$. These findings clearly emphasize the need for using the lowest possible dose of olanzapine in the treatment of schizophrenia. As described above, use of the population pharmacokinetic model and the dopamine $D_{2}$ receptor occupancy estimation model could bring psychiatric practice closer to the goals of personalized medicine: enabling the establishment of individualized antipsychotic doses that would result in optimal $\mathrm{D}_{2}$ receptor occupancy and clinical response.

In this study, the authors used the collective data from 3 clinical trials and tested the validity and reliability of predicting plasma concentrations of olanzapine following a dosage change from baseline concentrations, using population pharmacokinetic techniques [20]. The predicted post-adjustment concentrations of olanzapine were compared to the actually measured concentrations in order to determine the reliability of our prediction strategy.

\section{Methods}

\section{$\nabla$}

\section{Subjects and settings}

Data used for the present analysis were derived from 3 clinical trials; in those 3 trials, plasma olanzapine concentrations were measured twice before a change in dosage and once after the change. The first study was designed specifically for the present analysis. In this first study, male or female patients of any race who fulfilled the following criteria were included: (1) diagnosis of schizophrenia, schizoaffective disorder, schizophreniform disorder, delusional disorder, or psychotic disorder not otherwise specified according to the Diagnostic and Statistical Manual of Mental Disorders, Fourth Edition [21], (2) having changed a dose due to insufficient treatment response or side effect, and (3) having been treated with oral tablet form of olanzapine at a steady dose for at least 7 days. The study was conducted at Keio University Hospital, Minami-Hannou Hospital, and Asakadai Mental Clinic and was approved by the institutional review board at all participating sites. The study subjects provided written informed consent after receiving detailed information about the protocol. The trial was registered at the University Hospital Medical Information Network (UMIN) Clinical Trials Registry as UMIN000002294. The second study was an open-label, 28-week, randomized controlled trial to investigate the impact of olanzapine or risperidone dose reduction by half on cognitive function in stable patients with schizophrenia. In this study, clinically stable patients with schizophrenia receiving a stable dose of either olanzapine $>5 \mathrm{mg}$ /day or risperidone $>2 \mathrm{mg} /$ day as antipsychotic monotherapy for at least 3 months were included [22] (UMIN Clinical Trials Registry, UMIN000001834). In the third study, patients aged 50 and older with schizophrenia spectrum disorders who had been treated with olanzapine at a steady dose of $\geq 10 \mathrm{mg}$ /day or risperidone at a steady dose of $\geq 2 \mathrm{mg}$ /day for 6-12 months were included [23] (Clinicaltrials.gov, NCT00716755). This study included PET scanning to measure dopamine $\mathrm{D}_{2}$ receptor occupancy before and after a gradual dose reduction of antipsychotics by up to $40 \%$.

\section{Study description}

The design of the present analysis is summarized in $\bullet$ Fig. 1. 2 plasma samples were taken at 2 different random time points for the measurement of plasma olanzapine concentrations. At least 7 days after a dosage change of olanzapine, the third sample was collected at any given time point.

The plasma olanzapine concentration of the third sample was kept blind to the investigator (R.R.B.), who calculated the predicted plasma concentrations using NONMEM VII [24]. Plasma concentrations of olanzapine were assayed in heparinized plasma using liquid chromatography with tandem mass spectrometry at the Centre for Addiction and Mental Health, Toronto, Canada. The following information was also collected: concomitant medications, intervals between last dose and blood draw, and demographic and clinical information, including age, diagnosis, sex, race, and smoking status. All subjects recorded the time of their last dose before the scheduled collection of blood samples, and concomitant medications were kept constant throughout the study period. In all subjects, medication adherence was evaluated by their verbal report. We excluded the subjects who lacked any information needed for population pharmacokinetic analysis (e.g., dosing interval, weight).

\section{Population pharmacokinetic analysis}

Using a previously established population pharmacokinetic model for olanzapine [20], the plasma concentration of olanzapine at the time of the third blood draw (i.e., the blood draw after a dosage change) was individually predicted with the 2 plasma concentrations of olanzapine at the baseline dose, interval between last dose and blood draw, age, and smoking status ( $\bullet$ Fig. 1). The mixed-effects model was established using the data derived from the Clinical Antipsychotic Trials in Interven-

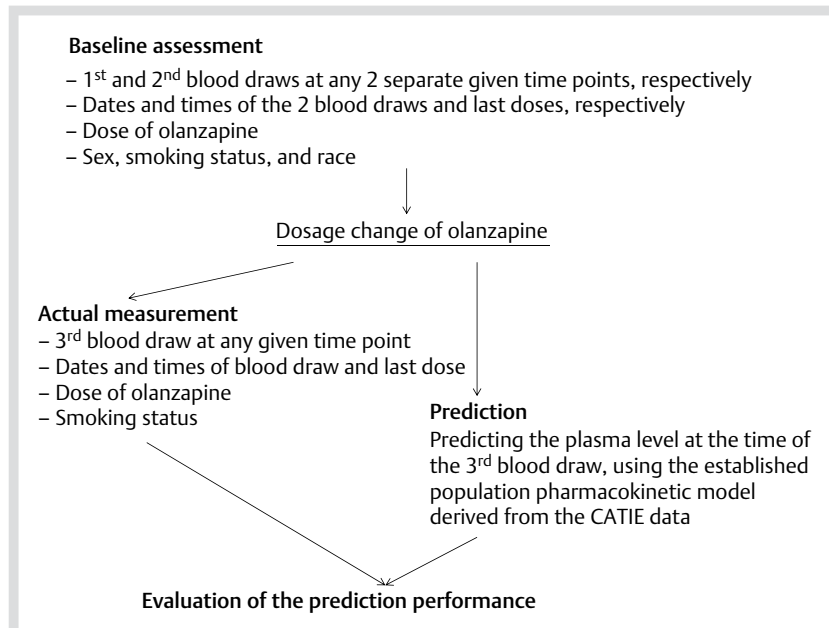

Fig. 1 Summary of the Study Design. 
tion Effectiveness (CATIE). This dataset included olanzapine concentrations (1527 plasma samples) from 523 subjects (age range, 18-103 years); a one-compartment mixture model with additive error best described the data [20]. According to the results of this trial, sex, race, and smoking habits that were found to affect olanzapine clearance were included in this population pharmacokinetic model for the present study.

\section{Statistical analysis}

Statistical analyses were carried out using the IBM SPSS Statistics Version 21 (IBM Corporation, Armonk, NY, USA) and the GraphPad Prism Version 5 (GraphPad Software, Inc, San Diego, CA, USA). The predictive performance of this procedure was assessed with the mean prediction error and the root-meansquared prediction error [25]. The prediction error refers to the difference between the true (i.e., measured) values and those predicted by the model. The mean squared prediction error is defined as a mean of squared values of prediction errors for all data points. The root-mean-squared prediction error refers to the root of the mean squared prediction error, which is a measure of "bias." Pearson correlation analysis was also used to examine the relationship between the observed and predicted values although this is a less precise value of predictive performance. A p-value of $<0.05$ was considered statistically significant (2-tailed).

\section{Results}

$\nabla$

Data from a total of 31 subjects ( 3 outpatients from the first study, 2 inpatients and 7 outpatients from the second study, and 19 outpatients from the third study) were used for the present analysis; their demographic and clinical characteristics are summarized in Table 1. The dose of olanzapine was increased and decreased in 2 and 29 subjects, respectively (mean \pm SD dose, $\mathrm{mg}$ /day: $5.0 \pm 0$ to $7.5 \pm 0$ and $18.1 \pm 7.7$ to $11.1 \pm 5.3$, respectively). Inter-individual variations in the oral clearance of olanzapine were as wide as approximately 4 -fold (range, $12-47 \mathrm{~L} / \mathrm{h}$ ).

The mean (95\% confidence interval) prediction errors and rootmean-squared prediction errors $(\mathrm{ng} / \mathrm{mL})$ were as low as 1.6 ( -2.8 to 6.0 ) and 12.0 (8.9 to 15.0 ), respectively ( $\bullet$ Fig. 2 ). The observed and predicted concentrations of olanzapine were highly correlated $(r=0.91, P<0.0001)$ ( $\odot$ Fig. 2$)$. The distribution of the prediction errors is shown in $\odot$ Fig. 3.

\section{Discussion}

$\nabla$

To the best of our knowledge, this is the first attempt to predict the plasma concentration of olanzapine following a dosage change in patients with schizophrenia spectrum disorders with the use of population pharmacokinetic techniques. We have previously reported that plasma concentrations of risperidone can be reliably predicted prior to a dose adjustment using the same population pharmacokinetic technique [9]. Consistent with our previous report, the present study demonstrates that the same methodology can also be applied to olanzapine in a reliable manner.

Considering that a model to estimate the dopamine $\mathrm{D}_{2}$ receptor occupancy from plasma concentrations of olanzapine is available [4], the results of this study can be used to predict peak and trough $\mathrm{D}_{2}$ receptor occupancy levels before dose adjustment of
Table 1 Demographic and Clinical Characteristics of Subjects $(N=31)$.

\begin{tabular}{|c|c|}
\hline Characteristics & Values \\
\hline Age, mean $\pm S D$ (range), years & $56.0 \pm 11.6(30-72)$ \\
\hline Male, N (\%) & $19(61.3 \%)$ \\
\hline Smoking, N (\%) & $10(32.3 \%)$ \\
\hline \multicolumn{2}{|l|}{ Race } \\
\hline Caucasian, N (\%) & $27(87.1 \%)$ \\
\hline African, N (\%) & $4(12.9 \%)$ \\
\hline Inpatient, N (\%) & $7(22.6 \%)$ \\
\hline \multicolumn{2}{|c|}{ Dose of olanzapine, mean \pm SD (range), $\mathrm{mg}$} \\
\hline Before dosage change & $17.2 \pm 8.1(3.8-35.0)$ \\
\hline After dosage change & $10.9 \pm 5.2(2.5-22.5)$ \\
\hline
\end{tabular}

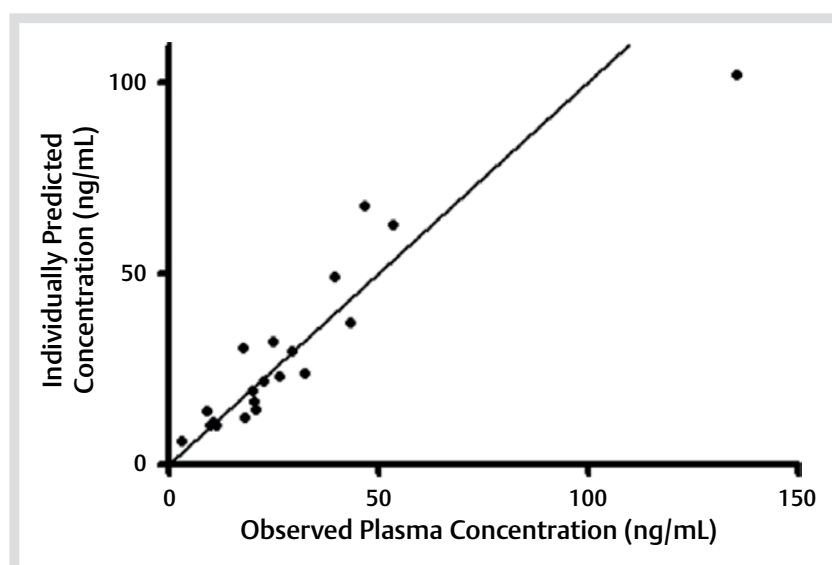

Fig. 2 Relationship between observed and predicted plasma concentrations of olanzapine, using population pharmacokinetic techniques.

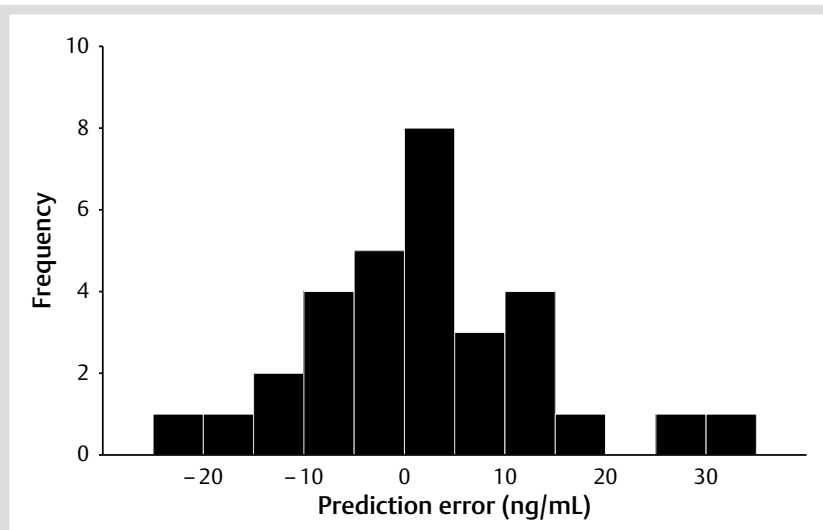

Fig. 3 Distribution of prediction errors.

olanzapine in individual patients without performing a brain imaging study. This is of high clinical pertinence for the treatment of schizophrenia, since excessive striatal $\mathrm{D}_{2}$ receptor blockade has consistently been associated with extrapyramidal side effects $[1,3,4]$. Furthermore, excessive dopamine $D_{2}$ receptor blockade also increases the risk of cognitive impairment [26] and hyperprolactinemia [27]. On the other hand, insufficient $D_{2}$ receptor blockade has been shown to be associated with poor clinical response in the acute phase of the illness [1,3]. These findings emphasize the importance of targeting an appropriate dopamine $\mathrm{D}_{2}$ receptor blockade of $65-80 \%$, the so-called "therapeutic window", in the acute phase of treatment for persons 
with schizophrenia. Moreover, physicians as well as patients are often reluctant to reduce the antipsychotic dose during the maintenance treatment of schizophrenia in fear of potential relapse. As a result, patients continue to be maintained on higher doses of antipsychotics than actually needed. If the lowest possible effective dose can be estimated for each individual, such reluctance will be dispelled and the situation will change for more individualized treatment.

Using widely available drug assays for 2 blood samples collected at any given time point, the population pharmacokinetic model could be used to determine a new dose resulting in estimated peak and trough drug concentrations that would correspond to the therapeutic window of dopamine $\mathrm{D}_{2}$ receptor occupancy. According to the model for the estimation of dopamine $\mathrm{D}_{2}$ receptor blockade from plasma antipsychotic concentrations, the therapeutic window of $65-80 \%$ corresponds to plasma concentrations of olanzapine of 13.9-43.6 ng/mL [5]. Similarly, when antipsychotic treatment is initiated, the minimal effective oral dose of olanzapine associated with the targeted dopamine $\mathrm{D}_{2}$ receptor occupancy could also be individually estimated. This can be accomplished by measuring the baseline plasma drug concentrations at steady state of a starting test dose of olanzapine, such as $2.5 \mathrm{mg}$ oral dose per day for 1 week. Thus, personalizing antipsychotic doses to ensure optimal clinical response based on estimated striatal $\mathrm{D}_{2}$ receptor occupancy may one day become common clinical practice; using plasma drug concentrations and population pharmacokinetic models [7].

While the utility of therapeutic drug monitoring (TDM) of olanzapine for the treatment of schizophrenia has still been controversial [28], a number of studies have suggested a link between its blood concentration and clinical outcomes [29-32]. For example, Fellows et al. identified a maximal plasma olanzapine concentration of $23 \mathrm{ng} / \mathrm{mL}$ as a cut-off point to distinguish responders from non-responders [29]. Moreover, according to Mauri et al., plasma olanzapine concentrations ranging from 5 to $120 \mathrm{ng} / \mathrm{m}$ showed a curvilinear correlation with clinical improvement in patients with schizophrenia [31]. Furthermore, using a large naturalistic dataset from an olanzapine TDM service, Patel and colleagues found that for a given dose of olanzapine female non-smokers had higher plasma concentrations than male smokers. This data suggests that TDM of olanzapine may be useful not only in assessing adherence, but also in limiting oral doses to reduce the risk of long-term toxicity [32]. In 2011, the Arbeitsgemeinschaft für Neuropsychopharmakologie und Pharmakopsychiatrie (AGNP) released the consensus guidelines for TDM of olanzapine, suggesting an optimal therapeutic range of plasma olanzapine concentrations between $20-80 \mathrm{ng} / \mathrm{mL}$ [33]. Notwithstanding such recommendations, TDM is rarely performed for olanzapine in clinical practice. Law et al. recently conducted an intervention study to test the clinical utility of TDM for olanzapine. They found that TDM could be feasibly implemented, potentially aiding dose optimization, supporting the routine clinical use of TDM-based dose adjustment for olanzapine [34]. To summarize, the evidence of TDM for olanzapine thus far indicates that measuring the plasma concentration of olanzapine seems to have the potential to guide physicians in determining its effective dose, especially in the face of insufficient response and dose-dependent side effects.

There are some limitations to the present study worth noting. First, although the predictive performance was assessed with the mean prediction error and the root-mean-squared prediction error [25], there was no other model to compare the accu- racy of the results. Second, if we sought for the individualized treatment with antipsychotics, it might have been ideal to include genetic information. Third, our conclusions cannot be generalized to antipsychotics other than olanzapine and risperidone, although population pharmacokinetic models have been established for other antipsychotics, including ziprasidone [35], clozapine [6], and perphenazine [36]. Fourth, the pharmacokinetics of olanzapine follows linear and dose-proportional effect within the approved dosage range [37]. However, it may be nonlinear and disproportionate beyond a certain plasma concentration of olanzapine. Fifth, since this sample size was small, the statistical power may not be sufficient to draw conclusions. The findings in the present study should be confirmed by future investigations with larger sample sizes. Finally, our proposed model-guided antipsychotic dosing still requires further validation. Therefore, we have been performing a series of clinical trials to test the reliability of our prediction model in estimating target doses in the treatment of schizophrenia.

In conclusion, the results of the present study demonstrate that plasma olanzapine concentrations can be reliably predicted prior to an actual change in dosage using population pharmacokinetic techniques. Considering the close relationship between plasma concentrations, dopamine $\mathrm{D}_{2}$ receptor occupancy, and clinical (psychotherapeutic and adverse) response, these findings suggest that individualized dosing with the measurement of antipsychotic plasma concentrations, instead of the conventional trial-and-error strategy, may become a clinical option for the treatment of schizophrenia.

\section{Author Contributions}

$\nabla$

Dr. Uchida led the $1^{\text {st }}$ study. Dr. Takeuchi led the $2^{\text {nd }}$ study. Dr. Mamo and Graff-Guerrero led the $3^{\text {rd }}$ study. Drs. Bies and Uchida designed the study. Drs. Tsuboi, Bies, and Uchida analyzed the data. Drs. Tsuboi and Uchida wrote the first draft of the manuscript. All authors have contributed to and approved the final manuscript.

\section{Funding}

$\nabla$

Japan Research Foundation for Clinical Pharmacology (HU); Ministry of Education, Culture, Sports, Science and Technology of Japan, Grant-in-Aid for Young Scientists-B, 22791140, (HU); US National Institute of Mental Health, RO1MH084886-01A2, (DM, AG-G, BP); Canadian Institutes of Health Research, MOP97946, (DM, AG-G, BP).

\section{Previous Presentation}

$\nabla$

Some of the data were presented at the $23^{\text {rd }}$ meeting of the Japanese Society of Clinical Neuropsychopharmacology, Okinawa, October 24, 2013.

\section{Acknowledgements}

$\nabla$

Ms. Wanna Mar for her assistance as a research coordinator for the $3^{\text {rd }}$ study. 


\section{Conflicts of Interest and Financial Disclosure}

$\nabla$

Dr. Tsuboi has received manuscript fees from Dainippon Sumitomo Pharma and speaker's honoraria from Eli Lilly, Tsumura, Yoshitomi Yakuhin, Dainippon Sumitomo Pharma, Kracie Pharma and Mitsubishi Tanabe Pharma within the past 2 years.

Dr. Bies has received NIH, CAMH, Lilly and Indiana University grant funding.

Dr. Suzuki has received manuscript fees or speaker's honoraria from Dainippon Sumitomo Pharma, Eli Lilly, Astellas Pharma, Novartis Pharma, and Meiji Seika Pharma within the past 2 years.

Dr. Takeuchi has received fellowship grants from the Canadian Institutes of Health Research (CIHR) and manuscript fees from Dainippon Sumitomo Pharma within the past 2 years.

Dr. Nakajima has received fellowship grants from the Canadian Institutes of Health Research (CIHR) and manuscript fees from Dainippon Sumitomo Pharma and Kyowa Hakko Kirin within the past 2 years.

Dr. Graff-Guerrero has received research support from the following external funding agencies: the CIHR, US National Institute of Health, OMHF, Brain and Behavior Research Foundation (BBRF), Mexico ICyTDF, CONACyT, W. Garfield Weston Foundation, Brain Canada and Ministry of Economic Development and Innovation of Ontario within the past 2 years.

Dr. Plitman has received funding from the Canada Graduate Scholarship.

Dr. Mimura has received grants or consultant fees from Eisai, Astellas Pharma, GlaxoSmithKline and Meiji, and received speaker's honoraria from Astellas Pharma, Dainippon Sumitomo Pharma, Eli Lilly, GlaxoSmithKline, Janssen Pharmaceutical, Meiji, Otsuka Pharmaceutical, Pfizer, and Yoshitomiyakuhin within the past 2 years.

Dr. Pollock receives research support from the National Institute of Health and the Canadian Institutes of Health Research. Within the past 5 years, he has been a member of the advisory board of Lundbeck Canada (final meeting was May 2009) and Forest Laboratories (final meeting was March 2008). Dr. Pollock has served one time as a consultant for Wyeth (October 2008). He was also a faculty member of the Lundbeck International Neuroscience Foundation (LINF) (final meeting was April 2010).

Dr. Uchida has received grants from Astellas Pharmaceutical, Eisai, Otsuka Pharmaceutical, GlaxoSmithKline, Shionogi, Dainippon-Sumitomo Pharma, Eli Lilly, Mochida Pharmaceutical, Meiji-Seika Pharma, and Yoshitomi Yakuhin and speaker's honoraria from Otsuka Pharmaceutical, Eli Lilly, Shionogi, GlaxoSmithKline, Yoshitomi Yakuhin, Dainippon-Sumitomo Pharma, Meiji-Seika Pharma, Abbvie, MSD, and Janssen Pharmaceutical within the past 3 years. Other authors have nothing to disclose. Dr. Tsuboi has received speaker's honoraria from Eli Lilly. Dr. Suzuki has received speaker's honoraria from Eli Lilly. Dr. Mimura has received speaker's honoraria from Eli Lilly. Dr. Uchida has received grants from Eli Lilly and speaker's honoraria from Eli Lilly. Other authors have nothing to disclose.

\section{Affiliations}

Department of Neuropsychiatry, Kyorin University School of Medicine, Tokyo, Japan

Department of Neuropsychiatry, Keio University School of Medicine, Tokyo, Japan

Division of Clinical Pharmacology, Indiana University School of Medicine, Indianapolis, IN, USA
${ }^{4}$ Indiana Clinical and Translational Sciences Institute, Indianapolis, IN, USA Geriatric Psychiatry Division, Centre for Addiction and Mental Health, Toronto, ON, Canada

Department of Psychiatry, Inokashira Hospital, Tokyo, Japan

Complex Mental Health Division, Centre for Addiction and Mental Health, Toronto, ON, Canada

${ }^{8}$ Department of Psychiatry, University of Toronto, Toronto, ON, Canada ${ }^{9}$ Campbell Family Mental Health Research Institute, Research Imaging Centre, Centre for Addiction and Mental Health, Toronto, ON, Canada

Department of Psychiatry, University of Malta, Valetta, Malta

\section{References}

1 Farde $L$, Nyberg $S$, Oxenstierna $G$ et al. Positron emission tomography studies on D2 and 5-HT2 receptor binding in risperidone-treated schizophrenic patients. J Clin Psychopharmacol 1995; 15: 19S-23S

2 Uchida H, Suzuki T, Mamo DC et al. Effects of age and age of onset on prescribed antipsychotic dose in schizophrenia spectrum disorders: a survey of 1,418 patients in Japan. Am J Geriatr Psychiatry 2008; 16: 584-593

3 Kapur S, Zipursky $R$, Jones $C$ et al. Relationship between dopamine $\mathrm{D}(2)$ occupancy, clinical response, and side effects: a double-blind PET study of first-episode schizophrenia. Am J Psychiatry 2000; 157: 514-520

4 Uchida H, Takeuchi H, Graff-Guerrero A et al. Dopamine D2 receptor occupancy and clinical effects: a systematic review and pooled analysis. J Clin Psychopharmacol 2011; 31: 497-502

5 Uchida $H$, Takeuchi $H$, Graff-Guerrero A et al. Predicting dopamine $\mathrm{D}(2)$ receptor occupancy from plasma levels of antipsychotic drugs: a systematic review and pooled analysis. J Clin Psychopharmacol 2011; 31: 318-325

$6 \mathrm{Ng} \mathrm{W}$, Uchida H, Ismail $\mathrm{Z}$ et al. Clozapine exposure and the impact of smoking and gender: a population pharmacokinetic study. Ther Drug Monit 2009; 31: 360-366

7 Uchida $H$, Pollock BG, Bies RR et al. Predicting age-specific dosing of antipsychotics. Clin Pharmacol Ther 2009; 86: 360-362

8 Nakajima S, Uchida $H$, Bies RR et al. Dopamine D2/3 receptor occupancy following dose reduction is predictable with minimal plasma antipsychotic concentrations: an open-label clinical trial. Schizophrenia Bull 2015; (in press)

9 Uchida H, Mamo DC, Pollock BG et al. Predicting plasma concentration of risperidone associated with dosage change: a population pharmacokinetic study. Ther Drug Monit 2012; 34: 182-187

10 Hollingworth SA, Siskind DJ, Nissen LM et al. Patterns of antipsychotic medication use in Australia 2002-2007. Aust N Z J Psychiatry 2010; 44: $372-377$

11 Weinbrenner S, Assion HJ, Stargardt T et al. Drug prescription patterns in schizophrenia outpatients: analysis of data from a German health insurance fund. Pharmacopsychiatry 2009; 42: 66-71

12 Lindenmayer JP, Khan A, Iskander A et al. A randomized controlled trial of olanzapine versus haloperidol in the treatment of primary negative symptoms and neurocognitive deficits in schizophrenia. J Clin Psychiatry 2007; 68: 368-379

13 Tollefson GD, Sanger TM, Beasley CM et al. A double-blind, controlled comparison of the novel antipsychotic olanzapine versus haloperidol or placebo on anxious and depressive symptoms accompanying schizophrenia. Biol Psychiatry 1998; 43: 803-810

14 Mizrahi $R$, Rusjan P, Agid $O$ et al. Adverse subjective experience with antipsychotics and its relationship to striatal and extrastriatal D2 receptors: a PET study in schizophrenia. Am J Psychiatry 2007; 164: 630-637

15 Ray WA, Chung CP, Murray KT et al. Atypical antipsychotic drugs and the risk of sudden cardiac death. N Engl J Med 2009; 360: 225-235

16 Kinon BJ, Volavka J, Stauffer $V$ et al. Standard and higher dose of olanzapine in patients with schizophrenia or schizoaffective disorder: a randomized, double-blind, fixed-dose study. J Clin Psychopharmacol 2008; 28: 392-400

17 Ulcickas Yood M, Delorenze GN, Quesenberry CP Jr et al. Association between second-generation antipsychotics and newly diagnosed treated diabetes mellitus: does the effect differ by dose? BMC Psychiatry 2011; 11: 197

18 Mitchell M, Riesenberg R, Bari MA et al. A double-blind, randomized trial to evaluate the pharmacokinetics and tolerability of 30 or 40 $\mathrm{mg} / \mathrm{d}$ oral olanzapine relative to $20 \mathrm{mg} / \mathrm{d}$ oral olanzapine in stable psychiatric subjects. Clin Ther 2006; 28: 881-892

19 Simon $V$, van Winkel $R$, De Hert $M$. Are weight gain and metabolic side effects of atypical antipsychotics dose dependent? A literature review. J Clin Psychiatry 2009; 70: 1041-1050 
20 Bigos KL, Pollock BG, Coley KC et al. Sex, race, and smoking impact olanzapine exposure. J Clin Pharmacol 2008; 48: 157-165

21 Association AP. Diagnostic and Statistical Manual of Mental Disorders. $4^{\text {th }}$ (ed.). Washington, DC: American Psychiatric Press; 1994

22 Takeuchi H, Suzuki T, Remington $G$ et al. Effects of risperidone and olanzapine dose reduction on cognitive function in stable patients with schizophrenia: an open-label, randomized, controlled, pilot study. Schizophr Bull 2013; 39: 993-998

23 Graff-Guerrero A, Rajji TK, Mulsant BH et al. Evaluation of antipsychotic dose reduction in late-life schizophrenia: a prospective dopamine D2/3 receptor occupancy study. JAMA Psychiatry 2015; 72: 927-934

24 Sheiner $L B$, Rosenberg $B$, Marathe $V V$. Estimation of population characteristics of pharmacokinetic parameters from routine clinical data. J Pharmacokinet Biopharm 1977; 5: 445-479

25 Sheiner LB, Beal SL. Some suggestions for measuring predictive performance. J Pharmacokinet Biopharm 1981; 9: 503-512

26 Sakurai H, Bies RR, Stroup ST et al. Dopamine D2 receptor occupancy and cognition in schizophrenia: analysis of the CATIE data. Schizophr Bull 2013; 39: 564-574

27 Tsuboi T, Bies RR, Suzuki T et al. Hyperprolactinemia and estimated dopamine D2 receptor occupancy in patients with schizophrenia: analysis of the CATIE data. Prog Neuropsychopharmacol Biol Psychiatry 2013; 45: 178-182

28 Bishara $D$, Olofinjana 0 , Sparshatt $A$ et al. Olanzapine: a systematic review and meta-regression of the relationships between dose, plasma concentration, receptor occupancy, and response. J Clin Psychopharmacol 2013; 33: 329-335
29 Fellows L, Ahmad F, Castle DJ et al. Investigation of target plasma concentration-effect relationships for olanzapine in schizophrenia. Ther Drug Monit 2003; 25: 682-689

30 Lane HY, Guo SC, Hwang TJ et al. Effects of olanzapine plasma concentrations on depressive symptoms in schizophrenia: a pilot study. J Clin Psychopharmacol 2002; 22: 530-532

31 Mauri MC, Steinhilber CP, Marino R et al. Clinical outcome and olanzapine plasma levels in acute schizophrenia. Eur Psychiatry 2005; 20 : 55-60

32 Patel MX, Bowskill S, Couchman L et al. Plasma olanzapine in relation to prescribed dose and other factors: data from a therapeutic drug monitoring service, 1999-2009. J Clin Psychopharmacol 2011; 31: 411-417

33 Hiemke C, Baumann P, Bergemann N et al. AGNP consensus guidelines for therapeutic drug monitoring in psychiatry: update 2011. Pharmacopsychiatry 2011; 44: 195-235

34 Law S, Gudbrandsen M, Magill $N$ et al. Olanzapine and risperidone plasma concentration therapeutic drug monitoring: A feasibility study. J Psychopharmacol 2015

35 Wessels AM, Bies RR, Pollock BG et al. Population pharmacokinetic modeling of ziprasidone in patients with schizophrenia from the CATIE study. J Clin Pharmacol 2011; 51: 1587-1591

36 Jin Y, Pollock BG, Coley $K$ et al. Population pharmacokinetics of perphenazine in schizophrenia patients from CATIE: impact of race and smoking. J Clin Pharmacol 2010; 50: 73-80

37 Callaghan JT, Bergstrom RF, Ptak LR et al. Olanzapine. Pharmacokinetic and pharmacodynamic profile. Clin Pharmacokinet 1999; 37: 177-193 